\title{
APLICACIÓN DE ANALISIS DE IMAGEN Y TECNOLOGÍA NIRS A LA EVALUACION DE LA POROSIDAD DE PLANCHAS, LÁMINAS Y TAPONES DE CORCHO Y SU RELACION CON LA CALIDAD INDUSTRIAL
}

\section{APPLICATION OF IMAGE ANALYSIS AND NIRS TECHNOLOGY TO THE EVALUATION OF THE POROSITY OF PLANKS, SHEETS AND CORK STOPPERS, AND ITS RELATION WITH THE INDUSTRIAL QUALITY}

\author{
Isabel Gómez-Sánchez ${ }^{1}$, José Luis García de Ceca ${ }^{2}$, Juan García-Olmo ${ }^{3}$, Leticia Lara-Buil ${ }^{4}$, \\ Rafael López-Luque ${ }^{5}$, Cristina Prades ${ }^{5,4}$
}

\section{RESUMEN}

La calidad del corcho es una de las cuestiones más relevantes para el sector corchero. Es un proceso complejo que requiere evaluar múltiples factores y manejar simultáneamente variables cuantitativas y cualitativas, en ocasiones, con un alto grado de subjetividad.

Se presentan dos objetivos, en primer lugar establecer la relación entre la calidad comercial del corcho y la porosidad medida mediante análisis de imagen en diferentes etapas de la transformación industrial (planchas, láminas y tapones), y en segundo lugar, evaluar el potencial de la tecnología NIRS como método de caracterización de la porosidad y, por tanto, de la calidad industrial. Para ello se ha utilizado un colectivo muestral formado por 479 planchas, 176 láminas y 90 tapones de corcho natural de una pieza, clasificados industrialmente en 4, 2 y 3 clases de calidad, respectivamente. El coeficiente de porosidad se evaluó aplicando técnicas de análisis de imagen, en dos y tres clases de color. Los espectros NIRS se obtuvieron en la sección transversal (planchas y tapones), en la sección tangencial (láminas y tapones) y en la sección radial (tapones), con un espectrofotómetro Foss NIRSystems 6500 SY II, mediante la modalidad de reflectancia remota.

El análisis estadístico de la relación entre calidad y coeficiente de porosidad, medido mediante análisis de imagen, permite discriminar dos clases de calidad para planchas y láminas y tres clases para tapones. La clasificación de la imagen en un mayor número de cluster mejora los resultados.

Las mejores calibraciones NIRS se obtuvieron para la porosidad medida en 3 clases de color, coincidiendo con los resultados obtenidos mediante análisis de imagen y permiten discriminar dos clases de calidad en planchas (refugo y corcho taponable), las dos clases en láminas $\left(\mathrm{R}^{2}=0,83 ; \mathrm{r}^{2}=0,78\right)$ y las tres clases en tapones $\left(\mathrm{R}^{2}=0,67 ; \mathrm{r}^{2}=0,53\right)$.

Debido a las dificultades de automatización del análisis de imagen en las primeras etapas de la transformación industrial, la tecnología NIRS podría ser una herramienta objetiva que permitiera evaluar la porosidad y diferenciar dos clases de calidad en planchas y láminas y tres clases en tapones.

Palabras Clave: Aspecto visual, espectroscopía, infrarrojo cercano, Coeficiente de Porosidad, Quercus suber, Industria del corcho.

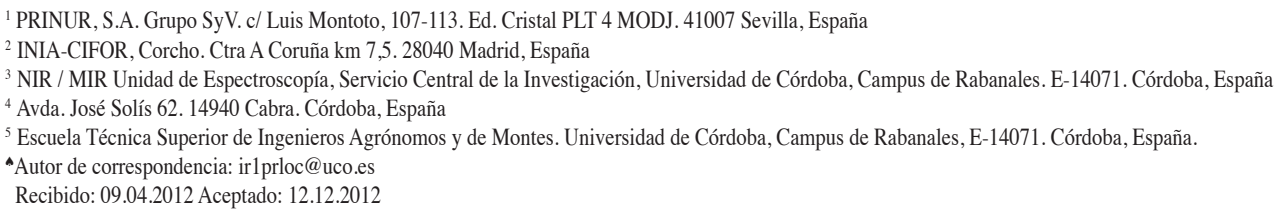




\begin{abstract}
The quality of cork is one of the most important issues for the cork industry. It is a complex process that requires evaluating many factors and simultaneously handles continuous and discrete variables, sometimes with a high degree of subjectivity.

There are two objectives, in first place to establish the relationship between the commercial quality of the cork and the porosity measured by image analysis at different stages of industrial processing (planks, sheets, and stoppers), and in second place, to evaluate the potential of NIRS technology as a method of assessing the porosity and, therefore, the industrial quality. For this, there has been used a sample group formed by 479 planks, 176 sheets and 90 one piece natural cork stoppers, classified industrially into 4, 2 and 3 quality classes, respectively. The coefficient of porosity was evaluated into two and three classes of color by using image analysis techniques. NIRS spectra were obtained in the transverse section (planks and stoppers), in the tangential section (sheets and stoppers) and in the radial section (stoppers), with a spectrophotometer Foss NIRSystems 6500 SY II, by remote reflectance mode.

Statistical analysis of the relationship between quality and coefficient of porosity, measured by image analysis, discriminates two quality classes for planks and sheets, and three classes for stoppers. Classifying the image into a larger number of clusters improves the results.

Best NIRS calibrations were obtained measuring the porosity into 3 classes of color, matching the results obtained by image analysis. This allow to discriminate two quality classes in planks (refuse and race), the two classes in sheets $\left(\mathrm{R}^{2}=0.83 ; \mathrm{r}^{2}=0.78\right)$ and the three classes for stoppers $\left(\mathrm{R}^{2}=0.67 ; \mathrm{r}^{2}=0.53\right)$.

Due to the difficulties of automated image analysis in the early stages of industrial processing, NIRS technology could be an objective tool to evaluate the porosity and to differentiate two classes of quality in planks and sheets, and three classes in stoppers.
\end{abstract}

Keywords: Visual aspect, near infrared, spectroscopy, Coefficient of Porosity, Quercus suber, cork industry

\title{
INTRODUCCIÓN
}

El corcho, o corteza del alcornoque (Quercus suber L.), es un material natural y renovable. La geometría de la célula y la composición química de su pared, así como la estructura del tejido lo convierten en un material singular apto para aplicaciones muy específicas derivadas de su alta elasticidad y sus propiedades aislantes. En su proceso de formación y crecimiento, el corcho está sujeto a una serie de alteraciones o anomalías, inherentes al propio corcho o provocadas por agentes externos bióticos y abióticos, que influyen en sus propiedades químicas, físicas y mecánicas, condicionando su utilización.

Se denominan planchas a las piezas extraídas del alcornoque que no han sufrido ninguna transformación exceptuando una cocción durante una hora y un recorte o dimensionamiento. Las planchas de corcho natural se utilizan para la fabricación del tapón natural y del disco natural. Los tapones naturales son cilindros rectos extraídos con una cuchilla cilíndrica de las planchas de corcho en dirección axial. Las láminas son una semimanufactura en el proceso de obtención del disco y se obtienen de las planchas mediante cortes paralelos con cuchilla circular, en dirección tangencial, y de anchura equivalente a la altura del disco. Los discos son cilindros rectos de corcho natural extraídos de las láminas en dirección radial, altura comprendida entre $3-6 \mathrm{~mm}$ y el mismo diámetro que el cuerpo del tapón, aglomerado o natural multipieza, al que se unirán (Figura 1).

En cada uno de estos procesos (planchas, láminas, tapones) se realiza un tipo de clasificación específico, en el que varían las unidades a clasificar, el número de clases y los criterios de asignación a cada clase. 
El factor que más influencia tiene en la calidad es la porosidad. La porosidad se debe a la presencia de los canales lenticulares, habitualmente denominados poros, que atraviesan el corcho en dirección radial permitiendo el intercambio gaseoso de los tejidos internos del árbol con el exterior. Se evalúa mediante el coeficiente de porosidad (CP) definido como el área de los canales lenticulares expresada en porcentaje del área total, entendiéndose en un sentido amplio cuando considera también la presencia y extensión de ciertos defectos, como fracturas o galerías ocasionadas por insectos (Pereira et al. 1994).

La clasificación de las planchas y las láminas se basa en un procedimiento que combina dos variables: El calibre o espesor de la plancha y el aspecto. En función del calibre, las planchas se destinan a la fabricación de tapones o, si el calibre es insuficiente, a laminación para la producción de discos. El aspecto se evalúa de manera visual por medio de operadores expertos, que tras examinar la plancha ó lámina, determinan la calidad de la misma sobre la base de su experiencia y sentido común. Este sistema de clasificación tradicional incorpora altos grados de subjetividad y variabilidad, pudiendo constatarse que las discrepancias entre clasificadores son mayores a medida que aumentan las clases de calidad. No existe una normativa específica que lo regule, por lo que cada fabricante establece el número de clases y los límites de ausencia/presencia de cada anomalía para cada clase de calidad (Barros y Pereira 1987, García de Ceca 2001, Lopes y Pereira 1998, Melo y Pinto 1989).

Al igual que en los casos anteriores no existen criterios normalizados para la clasificación del tapón. La clasificación se basa en el aspecto visual y se evalúa mediante técnicas de análisis de imagen del contorno del tapón (bases y costado) que miden el coeficiente de porosidad, y que a pesar de haber alcanzado un importante grado de desarrollo, requieren de una revisión manual. El análisis de imagen se realiza normalmente en dos clases de color, en blanco y negro o más recientemente en color (Costa y Pereira 2006, Costa y Pereira 2007, Vitria et al. 2007). Estas técnicas de momento no tienen aplicación industrial para planchas y láminas de corcho debido a la laboriosidad en su aplicación y la dificultad de su automatización (Benkirane et al. 2001, Gonzalez-Adrados et al. 2000, Pereira et al. 1996).

Otras técnicas aplicadas a la clasificación de los tapones son la tomografía Compton basada en los rayos X (Brunetti et al. 2002), la resonancia magnética nuclear en estado líquido ${ }^{1} \mathrm{H}$ MAS-NMR (Gil et al. 1997) y en estado sólido ${ }^{13} \mathrm{C}$-NMR (Hor et al. 2008, Neto et al. 1995, Pereira 2007) y la espectroscopía de infrarrojo medio FT-IR (Marques et al. 1994, Pereira 2007). Las técnicas ${ }^{13} \mathrm{C}-\mathrm{NMR}$ y FT-IR se han aplicado de forma combinada (Lopes et al. 2001) y recientemente el análisis de imagen se ha combinado con la espectroscopía de ondas en terahertz/ milímetros para la evaluación no destructiva de tapones de corcho (Hor et al. 2008).

La espectroscopía de infrarrojo cercano (NIRS) se ha aplicado a planchas de corcho en un estudio de viabilidad inicial que evalúa la relación entre los espectros NIR, la calidad tradicional y la porosidad del corcho sobre un colectivo de 170 muestras procedentes de Andalucía (España) y clasificadas en 8 clases de calidad mediante inspección visual. Las calibraciones cuantitativas proporcionaron coeficientes de determinación para validación cruzada de 0,47 para calidad visual y de 0,69 para porosidad, en la sección transversal, con un nivel de precisión que se aproxima a los resultados obtenidos mediante el método de referencia (Prades et al. 2010). Estos resultados muestran que la tecnología NIRS es una herramienta adecuada para mejorar el control de calidad del corcho, en combinación con otros instrumentos y controles manuales, que continuarán utilizándose considerando la heterogeneidad de un producto natural como el corcho. Se ha constatado también la aptitud de la tecnología NIRS para discriminar el origen geográfico del corcho (Prades et al. 2012).

Por todo ello, el objetivo de este trabajo es establecer la relación entre la calidad comercial del corcho y la porosidad, medida mediante análisis de imagen, y estudiar el potencial de la tecnología NIRS como método de evaluación de la porosidad e indirectamente como método de evaluación de la calidad industrial. Para corroborar y ampliar los resultados previos, el estudio se realizará para tres productos, planchas, láminas y tapones de corcho natural, incluyendo en el caso de las planchas, un mayor número de muestras que comprenda la variabilidad del corcho en lo que se refiere a calidades y procedencias. 
Debido a las dificultades de automatización del análisis de imagen en las primeras etapas de la transformación industrial, la tecnología NIRS podría ser la herramienta que permitiera la medición de la porosidad e introdujera un criterio objetivo en la clasificación comercial de planchas y láminas de corcho en la industria.

\section{MATERIALES Y MÉTODOS}

\section{Material experimental}

Se utilizaron tres colectivos independientes, corcho en plancha, láminas de corcho y tapones de corcho natural (Figura 1). La clasificación de calidades para cada colectivo fue realizada en las industrias de origen siguiendo el procedimiento tradicional (Tabla 1).

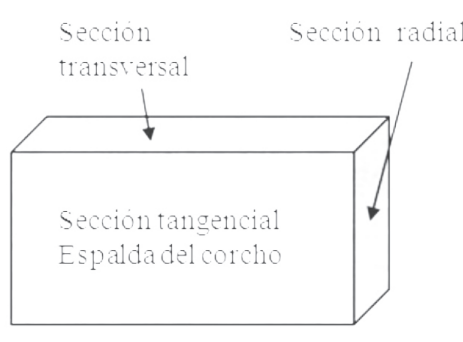

A

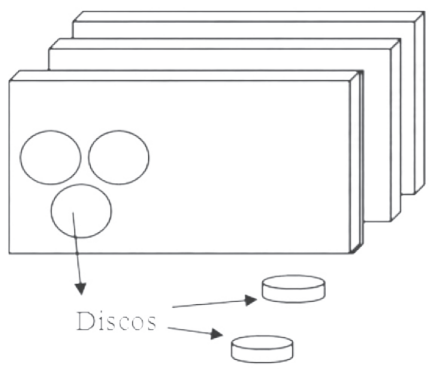

B.

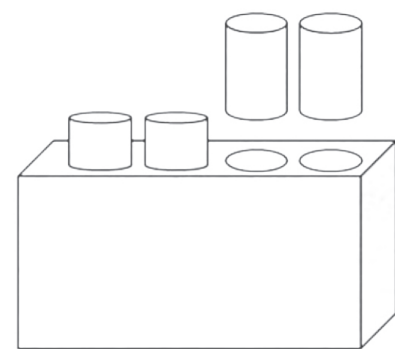

C

Figura 1. Esquema de las secciones de planchas, láminas y tapones

A. Planchas; B. Láminas; C. Tapones.

Tabla 1. Valores de Calidad para los tres colectivos muestrales.

\begin{tabular}{|l|c|c|c|c|c|}
\hline \multicolumn{7}{|c|}{ Calidad } \\
\hline Muestras & $\mathrm{N}$ & Alta & Media & Baja & Refugo \\
\hline Planchas & 439 & 122 & 101 & 112 & 104 \\
\hline Láminas & 176 & \multicolumn{2}{|c|}{108} & 68 & - \\
\hline Tapones & 90 & 30 & 30 & 30 & - \\
\hline
\end{tabular}

Las planchas de corcho proceden de la colección "CORKASSESS" y han sido facilitadas por el Centro de Investigaciones Forestales (CIFOR) del Instituto Nacional de Investigación y Tecnología Agraria y Alimentaria (INIA) de España. Provienen de los países con mayor superficie de alcornocal y con mayor producción de corcho, Marruecos (60 muestras), Portugal (200 planchas) y España (179 planchas).Se muestrearon las principales áreas productoras de cada país y todas las calidades se encuentran representadas. El colectivo está compuesto por 439 muestras con dimensiones aproximadas $20 \times 2 \mathrm{~cm}$ en las direcciones tangencial y axial, correspondiendo la dirección radial al espesor o calibre y siendo, en consecuencia, variable (Figura 1A). La clasificación de calidad del corcho en plancha se realizó por el método tradicional de inspección visual mediante la combinación de dos variables, calibre y calidad visual. Siguiendo el procedimiento industrial estándar, las muestras se cocieron en agua durante 1 hora y se clasificaron en origen en cuatro clases: alta, media, baja y refugo (corcho no apto para fabricar tapón). 
Las láminas de corcho natural se obtuvieron de 35 planchas de corcho cocido procedentes de Andalucía y de 2 calidades (20 planchas de calidad alta - media y 15 planchas de calidad baja) de dimensiones aproximadas 10x30 cm en las direcciones axial y tangencial (Figura 1B). Las planchas se laminaron a $1 \mathrm{~cm}$ de espesor en dirección radial, obteniéndose entre 1 y 4 láminas por plancha en función del calibre, la primera correspondía al vientre (superficie interior de la plancha que se separa del alcornoque en la extracción) y la última a la espalda (superficie exterior de la plancha). La primera lámina correspondiente al vientre tenía espesor variable debido al contorno irregular de la plancha. Únicamente las láminas centrales tenían ambas superficies limpias y $1 \mathrm{~cm}$ de espesor, pudiendo tener la lámina correspondiente a la espalda un espesor superior o inferior. Las láminas se cortaron con una cuchilla, en tantas submuestras como podían obtenerse de cada lámina y con dimensiones de 4,5 cm en dirección axial y $11,5 \mathrm{~cm}$ en dirección tangencial. Este tamaño fue seleccionado porque coincidía con el tamaño de la cápsula del instrumento NIRS que posteriormente se utilizaría para la obtención del espectro. Se identificó cada cara de la lámina con tres dígitos; el número de plancha, el número de lámina y una letra que indicaba su posición en relación al vientre del corcho. Las láminas se clasificaron en dos clases: Alta-media y baja, de acuerdo con la calidad industrial de las planchas de las que procedían.

El colectivo de tapones naturales estaba formado por 90 unidades procedentes de Andalucía (45 muestras) y Cataluña (45 muestras) de $24 \mathrm{~mm}$ de diámetro y $44 \mathrm{~mm}$ de longitud (Figura 1C). Se lijaron para obtener una superficie lisa, pero no fueron objeto de ningún otro tratamiento superficial. La clasificación industrial se realizó en tres clases: alta, media y baja.

Previamente a la obtención de los espectros las muestras de corcho en plancha y los tapones se estabilizaron en cámara climática marca Dycometal CCK 81 durante una semana a $20^{\circ} \mathrm{C}$ de temperatura y $65 \%$ de humedad relativa lo que equivale a una humedad de equilibrio higroscópico del corcho del $6 \%$.

\section{Porosidad}

La porosidad de las planchas y las láminas se evaluó mediante análisis colorimétrico de la apariencia, utilizando la metodología descrita en Prades et al. 2010.

La imagen digital de una cara plana de la muestra, obtenida mediante escaneado, se considera una matriz bidimensional en la que cada pixel cuenta con tres componentes de color. Las coordenadas de un píxel informan de su posición y sus tres valores del color (Rojo, Verde y Azul ó RGB) (Di Zenzo 1983). El escáner a utilizar debe estar calibrado para poder corregir los colores detectados y obtener los archivos de gráficos con el mismo formato pero con coordenadas RGB corregidas.

Para medir la superficie de poros dentro de una imagen, se utiliza el análisis cluster como un método de segmentación. En concreto, se utiliza el algoritmo K-medias (Pal y Pal 1993). Si la segmentación se realiza en dos clases o clusters, el algoritmo genera dos colores, uno oscuro para los poros y otro claro para las zonas tisulares. El algoritmo clasifica cada pixel de la imagen en claro u oscuro según la cercanía o mayor parecido a alguno de los dos colores propuestos. Dado que el tejido suberoso puede mostrar variaciones de color dentro de una misma muestra es posible que una clasificación basada en tres colores sea más acertada. En este caso, el algoritmo propone tres clases o colores como representativos de la imagen, uno oscuro para los poros y dos claros para el parénquima. Igualmente, el algoritmo clasifica cada pixel en cada una de las tres clases. En este caso se considerarán píxeles de poro aquellos que se clasifican como pertenecientes al color oscuro. El coeficiente de porosidad se estima como la proporción de píxeles clasificados como poros frente al total de píxeles.

En este trabajo la transformación de la imagen escaneada se realizó en 2 y 3 clases de color para las planchas y las láminas (Figura 2) y en dos colores para los tapones, asignando en todos los casos la tonalidad más oscura a los poros. 


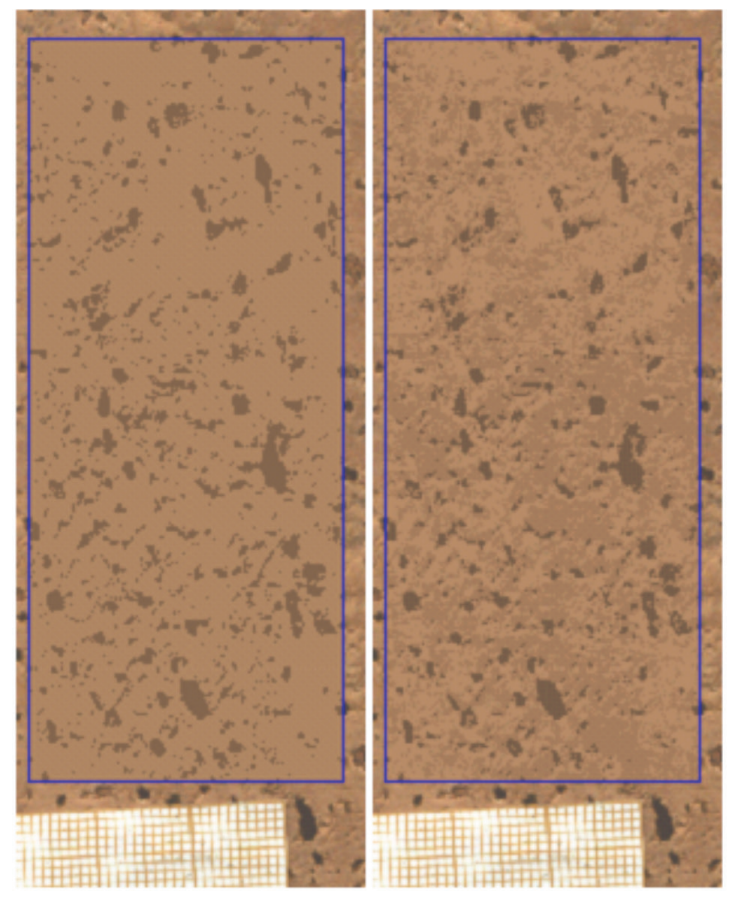

Figura 2. Análisis de imagen: A. Por2 B. Por3 en una lámina (muestra M1V).

El análisis de imagen de las planchas se realizó en la sección transversal y se obtuvieron dos mediciones por plancha, una a la izquierda y otra a la derecha. Se utilizó un tamaño de ventana de $4 \mathrm{~cm}$ en dirección tangencial y variable en dirección radial, coincidiendo con el calibre (Figura 1A). La porosidad de cada plancha se obtuvo como la media de ambas mediciones.

La porosidad de las láminas se obtuvo en la sección tangencial (Figura 1B). Se escanearon las caras limpias de todas las láminas, en una superficie de 4,5x11,5 cm, obteniéndose dos mediciones en las láminas centrales y vientre y una en la espalda. Se calculó la porosidad como la media de la porosidad obtenida en caras adyacentes de dos láminas consecutivas o en su defecto, como la porosidad de una única cara.

Para obtener la porosidad de los tapones se utilizó el programa Olympus Cell en dos ventanas diferentes. Para las bases de los tapones (sección transversal) se utilizó una plantilla circular de $20 \mathrm{~mm}$ de diámetro. Para el lateral se utilizó una plantilla rectangular de $10 \mathrm{~mm}$ x $40 \mathrm{~mm}$ en las secciones tangencial (paralela a los anillos de crecimiento) y radial (perpendicular a los anillos) (Figura 1C). La porosidad de las bases se obtuvo como la media de la porosidad de ambas bases y la porosidad del lateral se obtuvo como la media de las porosidades de las secciones tangencial y radial.

En resumen, se obtuvo el coeficiente de porosidad para:

- Sección transversal de las planchas en 2 clases de color

- Sección transversal de las planchas en 3 clases de color

- Sección tangencial de las láminas en 2 clases de color

- Sección tangencial de las láminas en 3 clases de color

- Secciones transversal de los tapones, en 2 clases de color

- Sección longitudinal (media de la sección tangencial y la radial) de los tapones en 2 clases de color 
El error típico de laboratorio (ETL) para la porosidad se calculó a partir de la siguiente fórmula:

$$
E T L=\sqrt{\frac{\sum_{i=1}^{N}\left(\mathrm{y}_{\mathrm{i} 1}-\mathrm{y}_{\mathrm{i} 2}\right)^{2}}{2 N}}
$$

donde $N$ es el número de muestras analizado e $y_{i 1}$ e $y_{i 2}$ son los dos valores obtenidos respectivamente a partir de las réplicas 1 y 2 de la muestra $i$.

Siendo para las planchas, $y_{i l}$ la porosidad de la zona izquierda e $y_{i 2}$ la porosidad de la zona derecha. Para las láminas, $y_{i l}$ la porosidad de la cara de una lámina e $y_{i 2}$ la porosidad de la cara adyacente de la siguiente lámina. Para las bases de los tapones $\mathrm{y}_{\mathrm{i} 1}$ es la porosidad de una de las bases e $y_{i 2}$ la porosidad de la otra base. Para los laterales de los tapones $y_{i 1}$ es la porosidad de la sección tangencial e $y_{i 2}$ la porosidad de la sección radial.

\section{Análisis Estadístico de la relación entre calidad y porosidad}

Con el objetivo de establecer las relaciones entre porosidad y calidad y analizar las diferencias entre los valores del coeficiente de porosidad, según la clase de color y la sección, se aplicó el siguiente tratamiento estadístico: Regresión simple, diagrama múltiple de caja y bigote, análisis de varianza de un solo factor y test de rango múltiple.

El análisis de regresión simple, mediante el modelo lineal $Y=a+b * X$, muestra si existe una relación estadísticamente significativa entre Por 2 vs. Por 3 , al nivel de confianza del $95 \%$.

El diagrama múltiple de caja y bigote se utiliza para comparar las muestras de Por 2 vs. Por 3 de las diferentes clases de calidad. El análisis incluye los nombres de la variable dependiente, la variable de factor y el número de observaciones y niveles en el análisis, por lo que facilita las comparaciones de la mediana, rango y valor extremo para cada grupo.

El análisis de varianza de un solo factor (ANOVA) determina el efecto de un factor cualitativo en una variable de respuesta. Muestra si existe una diferencia estadísticamente significativa entre la media de las variables Por2 vs. Por3 de un nivel de calidad a otro, para un nivel de confianza del $95 \%$.

El test de rango múltiple es un procedimiento de comparación múltiple para determinar qué medias son significativamente diferentes de las demás. Se utiliza para comparar las muestras de Porosidad_2 vs. Por 3 para los diferentes valores de calidad.

Los test se aplicaron a las planchas (Calidad 1, 2, 3, 4), láminas (Calidad 1,2) y tapones (Calidad 1, 2, 3).En el caso de las láminas, al estar clasificadas en dos calidades, el análisis de regresión incorpora el ANOVA, simplificándose el tratamiento estadístico.

\section{Instrumentación y recogida de espectros}

Las muestras se analizaron mediante un espectrofotómetro Foss-NIRSystems 6500 en la modalidad de reflectancia remota para planchas y tapones y reflectancia para láminas. Los datos de absorbancia de los espectros se obtuvieron en el rango 400-2500 nm, cada $2 \mathrm{~nm}$, como $\log 1 / \mathrm{R}$, donde R es la reflectancia. El rango $400-2500 \mathrm{~nm}$ comprende la región del visible VIS $(400-1098 \mathrm{~nm})$ y del infrarrojo cercano NIR $(1100-2500 \mathrm{~nm})$.

Debido a las diferentes presentaciones de las muestras, para el corcho en plancha y los tapones se utilizó una sonda de fibra óptica (NR-6539-A) y para las láminas una cápsula de 1⁄2 (Half cup).

$\mathrm{Al}$ variar sustancialmente los calibres de las planchas, el tamaño de la ventana para la recogida de espectros se ajustó con el objetivo de posibilitar la obtención de al menos dos espectros de cada muestra, uno a la derecha y otro a la izquierda, en la sección transversal. Se optó por utilizar la misma plantilla empleada para los tapones, con un 
tamaño de ventana de $10 \mathrm{~mm}$ x $40 \mathrm{~mm}$ y para ello se diseño un marco de plástico de poliestireno negro y se situó sobre la ventana original de la sonda NR-6539-A. Se recogieron de 2 a 10 espectros por muestra, en función de su calibre, para posteriormente mediarlos y obtener un espectro medio por muestra. A raíz del estudio previo realizado para el parámetro porosidad, en el que los resultados obtenidos para la sección tangencial fueron muy inferiores a los de la sección transversal, se ha trabajado con esta última sección (Prades et al. 2010).

Las láminas, de dimensiones 4,5x11,5 cm se introdujeron en la cápsula 1/2 (Half cup) del instrumento NIRS, obteniéndose el espectro en toda su superficie, correspondiente a la sección tangencial, y en ambas caras de la lámina. Se mediaron los espectros de las caras adyacentes de láminas consecutivas.

Para el tapón se utilizaron las mismas plantillas que para el análisis de imagen, circular de20 mm de diámetro para las bases y rectangular de 10 x 40mm para los laterales. Se obtuvieron 4 espectros por tapón: 2 espectros en las bases, uno en cada una, y 2 en el lateral, uno en la sección radial y otro en sección tangencial. Se mediaron los espectros de las bases y los espectros del lateral, obteniéndose 2 espectros medios por tapón, para los que se ajustarían posteriormente los modelos.

\section{Análisis quimiométrico cuantitativo}

Para la calibración y validación de la porosidad del corcho en plancha y tapones se utilizó como método de referencia el análisis de imagen en dos y tres clases de color. La recogida de espectros y el análisis quimiométrico cuantitativo se realizaron con el software WinISI II (Versión. 1.50) siguiendo la misma metodología de estudios previos (Prades et al. 2010, Prades et al. 2012).

Previamente a la calibración y para evaluar la calidad de los datos espectrales, se utilizó el algoritmo CENTER (Shenk y Westerhaus 1995) que realiza un Análisis de Componentes Principales (PCA) y calcula la distancia de cada uno de los espectros al centro del espacio definido por toda la población (distancia $\mathrm{H}$ de Mahalanobis), de tal forma que cuando esta distancia es superior a tres para una muestra determinada, el software la clasifica como anómala espectral (Shenk y Westerhaus 1995).

Para la obtención de las ecuaciones se utilizó el método de regresión por mínimos cuadrados parciales modificado (MPLS) y se estudió el uso de corrección scatter (corrección de la radiación dispersa) mediante el algoritmo Standard Normal Variate and Detrend (SNV\&DT). Las ecuaciones se desarrollaron en distintos rangos del espectro de 400$2500 \mathrm{~nm}$, aplicándose a los datos espectrales distintas combinaciones de tratamientos matemáticos de derivadas. En el programa WinISI los tratamientos matemáticos de derivadas se identifican mediante una notación de 4 dígitos $(a, b, c, d)$, donde a es el orden de magnitud de la derivada, b es el tamaño del segmento sobre el cual se realiza la derivación, c y d muestran el tamaño de los intervalos, expresados en nanómetros, empleados para el cálculo de suavización de la derivada.

Con el objetivo de incluir en esta etapa toda la variabilidad tanto espectral como química de los tres colectivos de calibración, se optó por validar las ecuaciones obtenidas mediante validación externa. La población total de espectros de dividió de forma aleatoria en dos colectivos, de calibración y validación, con $2 / 3$ y 1/3 de las muestras respectivamente.

Las mejores ecuaciones se seleccionaron en función del menor valor del error típico de validación cruzada (ETVC), el mayor valor del coeficiente de determinación $\left(\mathrm{r}^{2}\right)$ y el coeficiente de variación $(\mathrm{CV})$, calculado a partir del valor de ETVC y media del colectivo de validación empleado, considerados como los mejores estadísticos para determinar la capacidad predictiva de una ecuación (Shenk y Westerhaus 1995). 


\section{RESULTADOS Y DISCUSIÓN}

\section{Caracterización del colectivo de muestras. Variabilidad de los resultados en función de la porosidad}

El colectivo muestral inicial estaba compuesto por 479 planchas, 176 láminas y 90 tapones.

En la tabla 2 figuran los valores del coeficiente de porosidad, en función de la sección analizada (Figura 1) y del número de clases de color utilizados en su estimación (Figura 2). Debido al proceso de manipulación y clasificación de las muestras, el número de láminas utilizado para la medición de la porosidad se redujo a 145.

Tabla 2. Valores del Coeficiente de Porosidad para los tres colectivos muestrales.

\begin{tabular}{|l|l|c|c|c|c|c|c|c|}
\hline \multicolumn{1}{|c|}{ Coeficiente de Porosidad CP (\%) } \\
\hline Colectivo & Sección & Colores & $\mathrm{N}$ & Rango & Media & DS & ETL & CV \\
\hline Planchas & Transversal & 3 & 479 & $1,45-26,25$ & 6,67 & 3,21 & 1,81 & 27,14 \\
\cline { 3 - 10 } & & 2 & 479 & $1,69-36,73$ & 10,61 & 5,34 & 3,08 & 29,03 \\
\hline \multirow{2}{*}{ Láminas } & Tangencial & 3 & 145 & $2,04-21,55$ & 9,80 & 4,16 & 1,32 & 13,47 \\
\cline { 5 - 10 } & & 2 & 145 & $3,97-39,12$ & 17,24 & 7,69 & 5,75 & 33,35 \\
\hline \multirow{2}{*}{ Tapones } & Transversal & \multirow{2}{*}{2} & 90 & $1,86-25,23$ & 8,23 & 4,22 & 0,63 & 7,65 \\
\cline { 2 - 7 } & Tangencial/Radial & & 90 & $0,27-29,90$ & 7,67 & 5,70 & 0,73 & 9,51 \\
\hline
\end{tabular}

DS: Desviación estándar; ETL: Error técnico de laboratorio; CV: Coeficiente de Variación (\%)

El coeficiente de porosidad para los tres colectivos varía en un rango amplio $(0,27 \%$ y $39,12 \%)$, con menor intervalo de variación entre los límites inferiores $(0,27$ y 3,97$)$ que en los superiores $(21,55$ y 39,12$)$, considerando los 2 métodos de medición. Este rango corresponde a la porosidad en 2 clases de color disminuyendo $(1,45 \mathrm{y}$ $26,25 \%$ ) para 3 clases de color. También el coeficiente de porosidad medio en 3 clases de color es menor que en 2 clases de color, tanto para planchas (6,67 frente a 10,61 \%) como para láminas (9,80 frente a 17,24 \%), variando la desviación estándar entre 3,21 y 4,16.

Al comparar las porosidades en función de las secciones del corcho, el rango para la sección transversal en planchas en 3 clases de color $(1,45-26,25 \%)$ y tapones $(1,86-25,23 \%)$ es muy similar, a pesar de que los tapones han experimentado un proceso de selección más exigente. Las mayores diferencias obtenidas entre los rangos de las secciones longitudinales de las láminas $(2,04-21,55 \%)$ y los tapones $(0,27-29,90 \%)$ pueden deberse a que la porosidad se obtiene para las láminas en la sección tangencial y para los tapones como media de las secciones tangencial y radial.

Los ETL obtenidos ponen de manifiesto diferencias en la clasificación, siendo mayores a 1 para planchas y láminas e inferiores a 1 para tapones, debido probablemente a su mayor homogeneidad (Tabla 2). El ETL de la porosidad en 2 clases de color es mayor que en 3, lo que indica que la medición en 2 clases de color, además de proporcionar mayores valores del coeficiente de porosidad, aporta mayores discrepancias en la clasificación. Sería conveniente utilizar la porosidad en 3 clases de color, que permite discriminar defectos o anomalías del corcho no relacionados con la porosidad y que son catalogados como tales en la clasificación de 2 clases de color.

\section{Relación entre calidad y porosidad}

El análisis de regresión simple (Tabla 3) muestra valores del coeficiente de correlación, Por2 vs. Por3, similares para el conjunto de planchas y láminas de 0,77 y 0,67 respectivamente. Al considerar la clase de calidad, el coeficiente de correlación varía entre 0,67 y 0,89 en todos los casos, excepto para la calidad 1 de planchas $(0,47)$ y láminas $(0,34)$, lo que indica que las medidas de porosidad difieren más entre sí cuanto mejor es la calidad de la materia prima (Tabla 3$)$. 
Tabla 3. Resultados del análisis de regresión simple. Correlación entre coeficientes de porosidad obtenidos por diferentes métodos (planchas y láminas) y en diferentes secciones (tapones), en función de la calidad.

\begin{tabular}{|l|c|c|c|}
\hline \multicolumn{5}{|c|}{ Calidad planchas: Por2 vs. Por3 } \\
\hline PLANCHAS & Regresión & Coef. correlación $r$ & $\mathrm{R}^{2}$ \\
\hline TODAS & Por3 $=1,71954+0,466868^{*}$ Por2 & 0,77 & 60,17 \\
\hline CALIDAD 1 & Por3 $=3,45548+0,265759^{*}$ Por2 & 0,47 & 22,05 \\
\hline CALIDAD 2 & Por3 $=3,1428+0,345127^{*}$ Por2 & 0,75 & 55,99 \\
\hline CALIDAD 3 & Por3 $=1,13438+0,581865^{*}$ Por2 & 0,89 & 79,13 \\
\hline REFUGO & Por3 $=1,26624+0,536485^{*}$ Por2 & 0,80 & 64,85 \\
\hline \multicolumn{5}{|c|}{ Calidad láminas: Por2 vs. Por3 } \\
\hline LÁMINAS & Regresión & Coef. Correlación $r$ & $\mathrm{R}^{2}$ \\
\hline TODAS & Por3 $=3,59085+0,360207^{*}$ Por2 & 0,67 & 44,02 \\
\hline CALIDAD 1 & Por3 $=5,02845+0,159053^{*}$ Por2 & 0,34 & 12,19 \\
\hline CALIDAD 2 & Por3 $=3,44079+0,408788^{*}$ Por2 & 0,74 & 53,95 \\
\hline \multicolumn{5}{|c|}{ Calidad tapones: PorB vs. PorL } \\
\hline TAPONES & Regresión & Coef. Correlación $r$ & $\mathrm{R}^{2}$ \\
\hline TODAS & PorB $=-0,440101+0,985911^{*}$ PorL & 0,73 & 52,83 \\
\hline CALIDAD 1 & PorB $=-0,36933+0,803451^{*}$ PorL & 0,76 & 57,13 \\
\hline CALIDAD 2 & PorB $=0,605338+0,850227^{*}$ PorL & 0,67 & 42,41 \\
\hline CALIDAD 3 & PorB $=1,35976+0,918318^{*}$ PorL & 0,63 & 38,30 \\
\hline
\end{tabular}

El grado de correlación confirma la viabilidad de la utilización de las técnicas de análisis de imagen para evaluar la porosidad por ambos métodos, aunque la clasificación de la imagen en un mayor número de cluster mejora los resultados. La porosidad con 3 clases de color permite discriminar defectos o anomalías del corcho no relacionados con la porosidad y que son catalogados como tales en la clasificación de 2 clases de color. La medición en 2 clases de color, además de proporcionar mayores valores del coeficiente de porosidad, aporta mayores discrepancias en la clasificación. En la práctica, las técnicas inicialmente aplicadas en la industria manejan dos cluster en blanco y negro y han evolucionado hacia técnicas que aplican escalas de colores (Costa y Pereira 2007).

Los valores del coeficiente de correlación PorB vs. PorL en tapones son también similares (entre 0,63 y 0,73) (Tabla 2.C). Dado que el valor de $P$ en la tabla ANOVA es inferior a 0,01 , existe una relación estadísticamente significativa entre PorB vs. PorL al nivel de confianza del $95 \%$, aunque la tabla de ANOVA muestra que no hay significación para el corte con la ordenada (valor de $\mathrm{p}-1=0,6638$; valor de $\mathrm{p}-2=0,7136$; valor de $\mathrm{p}-3=0,5762$ ).

La discriminación de calidades en función del coeficiente de porosidad, proporciona resultados similares, independientemente del método de estimación de la porosidad empleado, en 2 o 3 clases de color, tanto para planchas (Figura 3A, Figura 3B) como para láminas (Figura 3C, Figura 3D). 


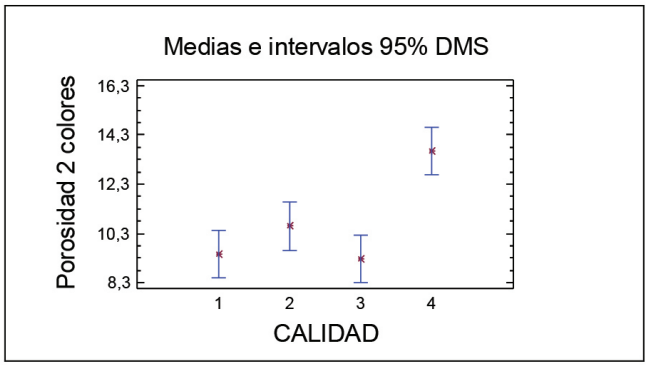

A. Planchas: Calidad v. Por 2

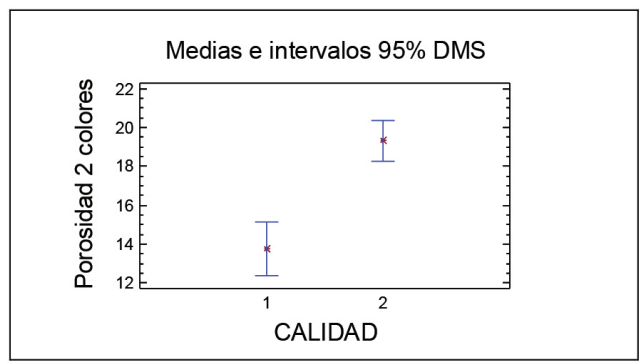

C. Láminas: Calidad vs. Por2

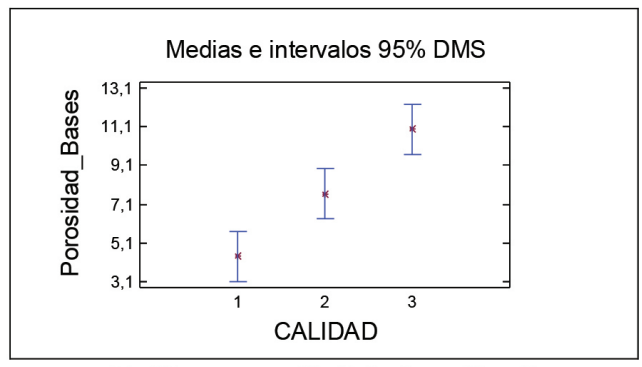

E. Tapones: Calidad vs. PorB

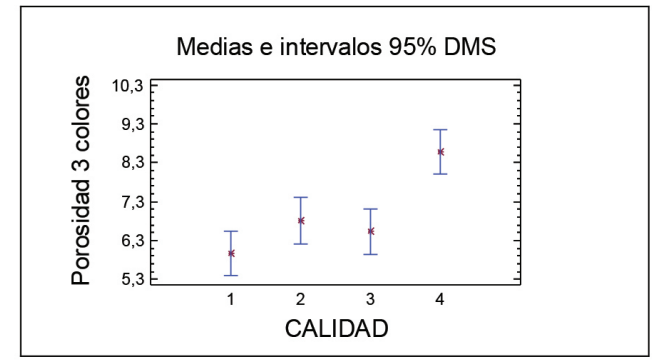

B. Planchas: Calidad vs. Por 3

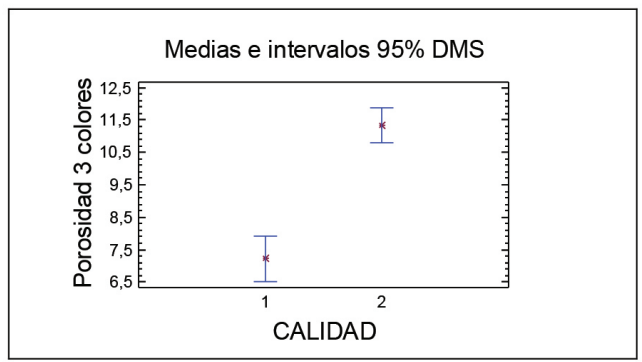

D. Láminas: Calidad vs. Por 3

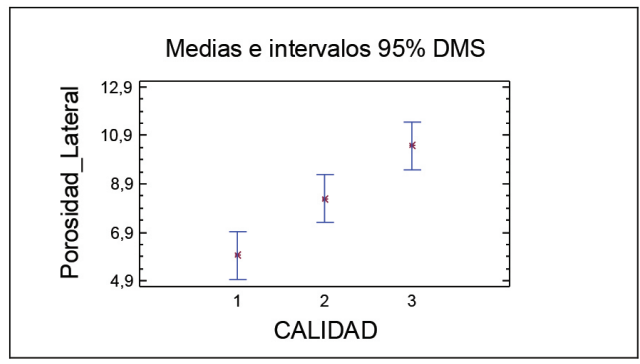

F. Tapones: Calidad vs. PorL

Figura 3. Diagrama múltiple de caja y bigote de la calidad en función del coeficiente de porosidad para planchas, láminas y tapones.

Para las planchas, está técnica permite clasificar las calidades extremas pero no establece diferencias entre calidades intermedias, tanto para 2 como para 3 clases de color. Existen diferencias estadísticamente significativas, a un nivel de confianza del $95 \%$, entre la calidad 4 y el resto de calidades, lo que confirma el análisis de varianza ANOVA, sin embargo, no se detectan diferencias significativas entre las calidades 1, 2, y 3 (Figura 3.A, Figura 3.B.).

En el caso de las láminas, existen diferencias estadísticamente significativas al nivel de confianza del $95 \%$ entre la calidad 1 y la calidad 2, para 2 y 3 clases de color, lo que también se confirma en el ANOVA (Figura 3.C, Figura 3.D). La diferenciación de las láminas es confiable, aunque en este resultado puede influir que se hayan considerado únicamente dos clases de cualidades.

Para los tapones, dado que el valor de $\mathrm{P}$ en la tabla ANOVA es inferior a 0,01 , existe una relación estadísticamente significativa entre PorB vs. PorL al nivel de confianza del $95 \%$, aunque no hay significación para el corte con la ordenada (valor de $\mathrm{p}-1=0,6638$; valor de $\mathrm{p}-2=0,7136$; valor de $\mathrm{p}-3=0,5762$ ). Al mismo tiempo, el coeficiente de curtosis indica la existencia de una no-normalidad significativa en los datos, lo que viola el supuesto de que proceden de distribuciones normales. Por lo tanto, se utiliza el test de Kruskal-Wallis para comparar las medianas en vez de las medias. Las tres clases de calidad se diferencian estadísticamente en función de la porosidad de las bases (Figura 3.E), coincidiendo con (Costa y Pereira 2005),y existe una diferencia significativa entre las calidades 1 y 3 , a partir de la porosidad de los laterales de los tapones (Figura 3.F). Este resultado podría mejorar calculando 
por separado la porosidad en las secciones tangencial y radial, sin embargo, se optó por mediar ambas secciones por razones prácticas.

\section{Estudio espectral}

Los espectros medios obtenidos para las planchas, láminas, bases y cuerpos de los tapones siguen un perfil similar, tanto en la región del visible (Figura 4A) como en la región NIRS (Figura 4B). Todos los espectros medios muestran las mismas bandas de absorción. Los espectros de las planchas y laterales son bastante parecidos y el espectro medio de las bases tiene menor intensidad de absorción $-\log (1 / \mathrm{R})$. Estas variaciones tienen su origen en las diferentes medidas de las ventanas y secciones del corcho utilizadas en cada caso (Prades et al. 2012). El espectro medio de las láminas se sitúa entre ambos, y aunque la superficie de absorción es muy superior $\left(51,75 \mathrm{~cm}^{2}\right)$ y la sonda utilizada es diferente, las bandas de absorción son las mismas.

Los picos de absorción NIR corresponden a las bandas 1215, 1450, 1750, 1930, 2150, 2300 y $2360 \mathrm{~nm}$. Estas bandas se asocian a los grupos-CH $(1215,1750,2150,2300$ y $2360 \mathrm{~nm}$ ) y grupo -OH (1450 y1930 nm) (Prades et al. 2010, Prades et al. 2012, Shenk et al. 2008). En la región VIS los espectros muestran la absorción máxima a $450 \mathrm{~nm}$.

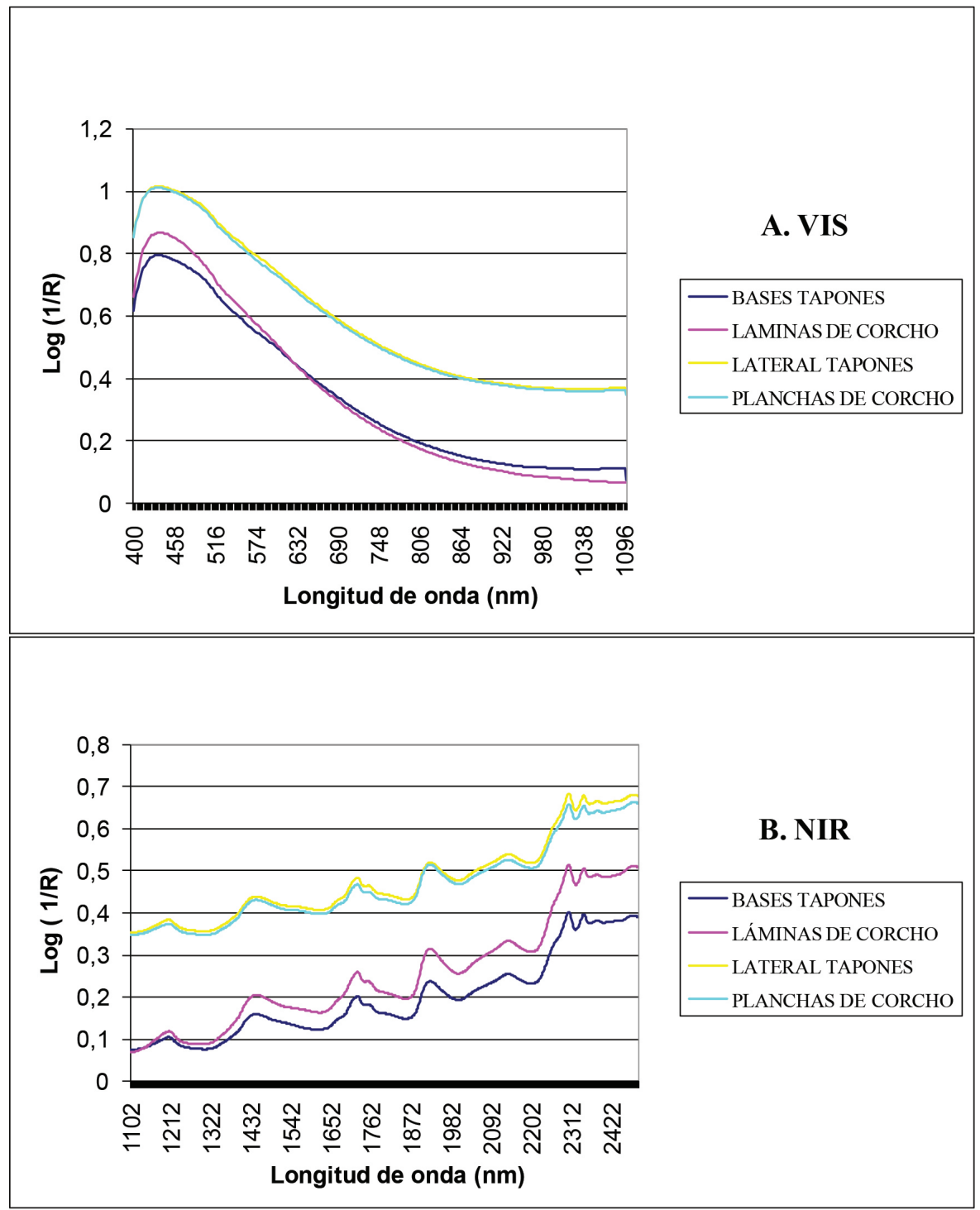

Figura 4. Espectro medio: Planchas, láminas, tapones. 


\section{Estudio de los modelos de predicción}

Para la calibración NIRS se utilizaron los datos del coeficiente de porosidad obtenidos para 3 clases de color debido a que presentaban un menor coeficiente de variación. Se seleccionaron los modelos que presentaban menores errores estándar de calibración y validación cruzada para los tres colectivos (Tabla 4). Los mejores resultados se obtuvieron para diferentes rangos del espectro y diferentes pretratamientos y únicamente para la base de los tapones la corrección SNV+DT mejoró la ecuación. Los coeficientes de determinación de la calibración varían entre 0,47 para las planchas y 0,83 para las láminas, siendo intermedios para ambas secciones de los tapones.

Tabla 4. Resultados del análisis cuantitativo NIRS de los 3 colectivos, planchas láminas y tapones, para el coeficiente de porosidad.

\begin{tabular}{|l|c|c|c|c|}
\hline \multicolumn{5}{|c|}{ Calibración del Coeficiente de Porosidad (CP) } \\
\hline COLECTIVO & 320 planchas & 99 láminas & \multicolumn{2}{c|}{60 tapones } \\
\hline PARÁMETRO & Por3 & Por3 & PorB & PorL \\
\hline SECCIÓN & Transversal & Tangencial & Transversal & $\begin{array}{c}\text { Tangencial/ } \\
\text { Radial }\end{array}$ \\
\hline \multirow{2}{*}{ LONGITUD ONDA } & $1100-2500 \mathrm{~nm}$ & $\begin{array}{c}400-2500 \\
\mathrm{~nm}\end{array}$ & $1100-2500 \mathrm{~nm}$ & $400-2200 \mathrm{~nm}$ \\
\hline CORRECCIÓN & NONE & NONE & SNV+D & NONE \\
\hline PRETRAT & $0,0,1,1$ & $2,5,5,1$ & $1,4,4,1$ & $1,4,4,1$ \\
\hline N & 297 & 96 & 57 & 59 \\
\hline MEDIA & 6,14 & 10,12 & 7,39 & 8,13 \\
\hline RANGO & $1,45-15,39$ & $3,06-21,55$ & $0,27-29,90$ & $2,24-22,66$ \\
\hline DT & 2,36 & 4,13 & 5,80 & 3,97 \\
\hline ETVC & 1,87 & 1,69 & 3,35 & 2,62 \\
\hline R $^{2}$ & 0,478 & 0,83 & 0,67 & 0,56 \\
\hline CV & 30,40 & 16,67 & 45,30 & 32,20 \\
\hline \multicolumn{5}{|c|}{ Validación del Coeficiente de Porosidad (CP) } \\
\hline PARÁMETRO & 159 planchas & 46 láminas & & 30 tapones \\
\cline { 2 - 6 } & Por3 & Por3 & PorB & PorL \\
\hline ETP & 1,77 & 1,98 & 3,82 & 4,77 \\
\hline N & 159 & 46 & 30 & 30 \\
\hline BIAS & 0,31 & $-0,52$ & $-0,46$ & $-0,27$ \\
\hline RANGO & $1,95-15,35$ & $2,04-16,33$ & $0,49-24,76$ & $3,23-25,23$ \\
\hline PENDIENTE & 0,82 & 1,09 & 0,87 & 0,45 \\
\hline${ }^{2}$ & 0,44 & 0,78 & 0,53 & 0,16 \\
\hline CV & 27,20 & 21,56 & 50,60 & 56,10 \\
\hline
\end{tabular}

Para las planchas la mejor ecuación se obtuvo en la región NIRS con tratamiento $0,0,1,1$ y sin corrección de dispersión. El menor coeficiente de determinación, en relación a los otros productos y a estudios previos (Prades et al. 2010) pueden deberse a la presencia de un mayor número de defectos y heterogeneidad en las planchas, con un CV próximo al 30\% (Tabla 4). Sin embargo, la precisión de la ecuación puede considerarse buena ya que los valores de ETL $(1,81)$ y ETVC $(1,86)$ son similares y los valores del CV del método de referencia $(27,14 \%)$ y de la calibración (30,40\%) también lo son. Los resultados obtenidos en la validación externa (Tabla 4) corroboran la bondad de la ecuación, adecuada para separar el refugo del corcho taponable. 
Para las láminas la mejor ecuación se obtuvo para todo el rango del espectro, sin corrección y tratamiento 2,5,5,1. El vientre del corcho, correspondiente a la sección tangencial en contacto con el árbol y en su forma original, había sido objeto de estudio con resultados bastante inferiores para planchas $\left(\mathrm{R}^{2}=0,39 ; \mathrm{ETVC}=2,61\right)$ (Prades et al. 2010). En este caso, la superficie limpia y sin curvaturas, el mayor tamaño de la ventana y la mayor precisión en la sonda utilizada proporcionan $\mathrm{R}^{2}=0,83$ y ETVC $=1,687$ algo superior al ETL $=1,32$ (Tabla 4). Análogamente al caso anterior, los valores del CV del método de referencia $(13,47 \%)$ y de la calibración $(16,67 \%)$ son similares y la validación externa confirma los resultados $\left(\mathrm{r}^{2}=0,78\right.$ y ETPC $\left.=1,93\right)$. La precisión de la ecuación puede considerarse buena (Tabla 4) (Shenk y Westerhaus 1996).

Para los tapones se obtuvieron mejores resultados en la sección transversal, sección que se recomienda para predecir la porosidad. La mejor ecuación $\left(\mathrm{R}^{2}=0,67\right.$ y ETVC $\left.=3,35\right)$ corresponde a la región NIRS, con tratamiento 1,4,4,1 y corrección SNV+DT (Tabla 4). Estos resultados mejoran los obtenidos en la misma sección y con la misma sonda para las planchas, confirmando la influencia del mayor grado de selección a que han sido sometidos los tapones. El coeficiente de determinación de la validación externa $\left(\mathrm{r}^{2}=0,53\right)$ indica que la ecuación es adecuada para discriminar 3 clases de calidad, entre valores bajos, medios y altos (Shenk y Westerhaus 1995), coincidiendo con los resultados obtenidos mediante el método de referencia (Costa y Pereira 2005, Costa y Pereira 2007). Sin embargo, el ETL = 0,63 es en este caso bastante inferior al ETVC $=1,69$ y al ETPC $=1,93$, lo que puede ser debido al menor número de muestras y a la no normalidad de los datos (Tabla 4).También sería necesario contrastar estos resultados utilizando el mismo software empleado para el cálculo del coeficiente de porosidad en planchas y láminas y comprobar la influencia del tamaño de la ventana en la obtención del espectro NIR, mediante otro equipo donde la ventana de medida fuera menor.

Teniendo en cuenta que la tecnología NIRS se emplea en este trabajo como método secundario de estimación de la porosidad, el objetivo a lograr es que los errores de estimación del método primario (análisis de imagen) y del método secundario (NIRS), ETL y ETVC respectivamente, sean similares. En el desarrollo de ecuaciones NIRS es frecuente realizar una etapa de evaluación de los modelos desarrollados o validación externa. El error de predicción en validación externa (ETP) es directamente comparable al ETL y al ETVC. Estos estadísticos, ETL, ETVC y ETP, dependen del valor medio de los datos empleados, por lo que para realizar la comparación se utiliza el estadístico adimensional CV(\%). Mientras mayor sea la similitud entre los valores de CV del error de los datos de porosidad estimados mediante análisis de imagen y los valores de CV de los errores de predicción NIRS para dicho parámetro, mayor fiabilidad, exactitud y precisión presentarán las ecuaciones NIRS desarrolladas (Shenk y Westerhaus 1995, Shenk y Westerhaus 1996).

En el caso de las planchas los valores de ETL $(1,81)$, ETVC $(1,87)$ y ETP $(1,77)$ son similares, y los valores del CV obtenidos mediante análisis de imagen (27,14\%), en la calibración NIRS (30,40\%) y en la validación externa NIRS $(27,20 \%)$ también lo son. Ambas técnicas proporcionan una precisión similar en la estimación del coeficiente de porosidad. En el caso de las láminas, ETL $(1,32)$ es algo inferior a ETCV $(1,69)$ y ETP $(1,98)$, y análogamente, el CV del análisis de imagen (13,4\%) es también algo inferior al CV de las ecuaciones NIRS ( $16,67 \%$ en la calibración y $21,57 \%$ en la validación). Considerando los coeficientes de determinación de la calibración $\left(\mathrm{R}^{2}=0,83\right)$ y la validación $\left(\mathrm{r}^{2}=0,78\right)$, las ecuaciones NIRS permitirían estimar la porosidad con una fiabilidad algo inferior a la que proporciona el análisis de imagen. Sin embargo, al realizar el mismo análisis para tapones se comprueba que no se obtienen adecuadas predicciones NIRS para la porosidad, por motivos expuestos con anterioridad.

En la misma medida en que la porosidad medida con análisis de imagen es un indicador de la calidad industrial (Costa y Pereira 2006, González-Adrados et al. 2000), la tecnología NIRS como método indirecto de medición de la porosidad también puede serlo, aunque los errores en la estimación de la porosidad mediante el método de referencia se sumen a los errores en la calibración NIRS.

Los coeficientes de determinación de la validación externa $\left(\mathrm{r}^{2}\right)$ obtenidos con la tecnología NIRS permitirían, según Shenk y Westerhaus (1995), discriminar dos calidades en planchas $\left(\mathrm{r}^{2}=0,44\right)$, refugo y corcho taponable, dos calidades en láminas $\left(\mathrm{r}^{2}=0,78\right)$, y tres calidades en tapones, $\left(\mathrm{r}^{2}=0,53\right)$, coincidiendo con la clasificación comercial en los dos últimos casos. 
El análisis estadístico y ANOVA del análisis de imagen muestran que para las planchas existen diferencias estadísticamente significativas, entre la calidad 4 y el resto de calidades, lo que permitiría clasificar las calidades extremas y separar el refugo del corcho taponable. La diferenciación de las láminas es confiable, aunque en este resultado puede influir que se hayan considerado únicamente dos clases de calidad. Para los tapones, existe una diferencia significativa entre las tres clases en función de la porosidad de las bases.

La tecnología NIRS puede introducirse en la industria como una herramienta apta para evaluar la porosidad en planchas y láminas, y como método indirecto que proporcione simultáneamente información objetiva sobre la calidad industrial, diferenciando dos clases de calidad en planchas y láminas, y tres clases en tapones.

\section{CONCLUSIONES}

La discriminación de calidades en función del coeficiente de porosidad mediante análisis de imagen proporciona resultados similares independientemente del número de clusters empleados en la segmentación del algoritmo K-medias. Sin embargo, la clasificación de la imagen en tres clases de color mejora los resultados, por lo que se recomienda ampliar el número de cluster para la estimación del coeficiente de porosidad.

En el análisis NIRS, los espectros medios obtenidos para las planchas, láminas, bases y cuerpos de los tapones siguen un perfil similar y muestran las mismas bandas y picos de absorción NIR $(-\mathrm{CH},-\mathrm{OH})$ de los estudios previos (Prades et al. 2010, Prades et al. 2012, Shenk et al. 2008). Las calibraciones del coeficiente de porosidad proporcionan resultados satisfactorios, considerando que los errores (ETVC) y CV son similares a los del método de referencia, excepto para tapones, donde el ETL es bastante inferior al ETVC. La validación externa corrobora la bondad de la calibración en todos los casos.

La relación entre porosidad y clases de calidad coincide para las dos técnicas empleadas, análisis de imagen y NIRS, que permiten separar de manera confiable dos clases de calidad en planchas (refugo y corcho taponable) y láminas, y tres clases en tapones.

La utilización del análisis de imagen para determinar la porosidad como criterio clasificador de la calidad de corcho está ampliamente contrastada para tapones y discos y aunque mejora los resultados de la clasificación visual en planchas no se aplica en la industria debido a dificultades en su automatización (Gonzalez-Adrados et al. 2000). La tecnología NIRS puede introducirse en la industria como una herramienta apta para evaluar la porosidad en planchas y láminas, con una precisión similar a la obtenida mediante análisis de imagen, y como método indirecto que proporciona simultáneamente información objetiva sobre la calidad industrial, permitiendo clasificar los productos de corcho natural en dos clases para planchas y láminas y en tres clases para tapones. 


\section{AGRADECIMIENTOS}

Este estudio ha sido parcialmente financiado por el Proyecto: RTA 2006-00119-C02-00. Los datos NIR se obtuvieron utilizando el hardware y software proporcionado por la unidad NIRS del SCAI de la Universidad de Córdoba. Agradecemos a la Dra. Ana Garrido Varo (E.T.S.I.A.M., Universidad of Córdoba, España) su apoyo en la realización de este trabajo.

\section{BIBLIOGRAFÍA}

Barros, I.; Pereira, H. 1987. Influencia do operador na classificaçao manual da cortiça por classes de qualidade. Cortiça 582: 103-105.

Benkirane, H.; Benslimane, R.; Hachmi, M; Sesbou, A. 2001. Possibilité de contrôle automatique de la qualité du liège par vision artificielle. Ann For Sci 58: 455-465.

Brunetti, A.; Cesareo, R.; Golosio, B.; Luciano, P.; Ruggero, A. 2002. Cork quality estimation by using Compton tomography. Nuclear Instruments and Methods in Physics Research B 196: 161 - 168.

Costa, A.; Pereira, H. 2005. Quality characterization of wine cork stoppers using computer vision. J Int Sci Vigne Vin 39(4): 209-218.

Costa, A.; Pereira, H. 2006. Decision rules for computer-vision quality classification of wine natural cork stoppers. American Journal of Enology and Viticulture 57(2): 210-219.

Costa, A.; Pereira, H. 2007. Influence of vision systems, black and white, colored and visual digitalization, in natural cork stopper quality estimation. Journal of the Science of Food and Agriculture 87(12): 2222-2228.

Di Zenzo, S. 1983. Advances in image segmentation. Image Vision Comput 1(4): 196-210.

García de Ceca, J.L. 2001. Factores que afectan a la calidad del corcho en planchas., in Dpto. Economía y Gestión de las Explotaciones e Industrias Forestales. Universidad Politécnica: Madrid.

Gil, A.M.; Lopes, M.; Rocha, J.; Neto, C.P. 1997. A 13C solid state nuclear magnetic resonance spectroscopic study of cork cell wall structure: The effect of suberin removal. International Journal of Biological Macromolecules 20: 293-305.

Gonzalez-Adrados, J.R.; Lopes, F.; Pereira, H. 2000. Quality grading of cork planks with classification models based on defect characterisation. Holz Als Roh-Und Werkstoff 58(1-2): 39-45.

Hor, Y.L.; Federici, J.F.; Wample, R.L. 2008. Nondestructive evaluation of cork enclosures using terahertz/ millimeter wave spectroscopy and imaging. Applied Optics 47(1): 72-78.

Lopes, F.; Pereira, H. 1998. Caracterizaao de planchas de cortica com diferente classificacao industrial. Sobreiro e Cortica. Conferencia Europeia sobre o Sobreiro e Cortica. Portugal: 332-340.

Lopes, M.H.; Barros, A.S.; Neto, C.P.; Rutledge, D.; Delgadillo, I.; Gil, A.M. 2001. Variability of cork from Portuguese Quercus suber studied by solid-state C-13-NMR and FTIR spectroscopies. Biopolymers 62(5): 268-277.

Marques, V.; Pereira, H.; Meier, D.; Faix, O. 1994. Quantitative-Analysis of Cork (Quercus suber L) and Milled Cork Lignin by FTIR Spectroscopy, Analytical Pyrolysis, and Total Hydrolysis. Holzforschung 48: 43-50. 
Melo, B.; Pinto, R. 1989. Análise de diferenças nos critérios de classificaçao qualitativa das rolhas. Cortiça 601: 293-302.

Neto, P.V.; Cardoso, A.L.; Gomes, C. 1995. Cork stoppers washing procedures. CTCOR Magazine 1(1): 20-23.

Pal, N.R.; Pal, S.K. 1993. A review on image segmentation techniques. Pattern Recognition 26(9): 1277-1294.

Pereira, H. 2007. Cork: Biology, Production and Uses. ed. Elsevier: Elsevier. 336p.

Pereira, H.; Lopes, F.; Graça, J. 1996. The evaluation of the quality of cork planks by Image Analysis. Holzforschung 50: 111-115.

Pereira, H.; Melo, B.; Pinto, R. 1994. Yield and quality in the production of cork stoppers. Holz als Roh und Werkstoff 52: 211-214.

Prades, C.; García-Olmo, J.; Romero, T.; García de Ceca, J.L.; López-Luque, R. 2010. Methodology for cork plank characterisation (Quercus suber L.) by near infrared spectroscopy and image analysis. Meas Sci Technol 21(065602): 1-11.

Prades, C.; Gómez-Sánchez, I.; García-Olmo, J.; González-Adrados, J.R. 2012. Discriminant analysis of geographical origin of cork planks and stoppers by near infrared spectroscopy. J Wood Chem Technol 32(1): 66-85.

Shenk, J.S.; Westerhaus, M.O. 1995. Routine Operation, Calibration, Development and Network System Management Manual. ed. N.S. Inc. Silver Spring, MD, USA.

Shenk, J.S.; Westerhaus, M.O. 1996. Calibration the ISI waqy., in Near Infrared Spectroscopy: The Future Waves.: Chischester, UK: 198-201.

Shenk, J.S.; Workman Jr, J.; Westerhaus, M.O. 2008. Application of NIR Spectroscopy to Agricultural Products., in Handbook of Near-Infrared Analysis, D.A. Burns and E.W. Ciurczak, Editors. CRC Press: Boca Raton FL, USA: $347-386$.

Vitria, J.; Bressan, M.; Radeva, P. 2007. Bayesian classification of cork stoppers using class-conditional independent component analysis. IEEE Transactions on Systems Man and Cybernetics Part C-Applications and Reviews 37(1): 32-38. 
